\section{Eating well}

Remarkably little is known about the signalling pathways that drive phagocytosis. Once its prey is bound to the cell surface, what exactly causes a phagocyte to extend pseudopodia around its dinner and swallow it? In the Journal of Cell Biology, Roberto Botelho and colleagues describe the spatial and temporal regulation of two signals involved in phagocytosis, providing both a visual and an intellectual feast.

The authors made use of fluorescent probes that specifically recognize membrane lipids. Using a macrophage cell line, they first turned their attention to the phospholipid phosphatidylinositol-4,5bisphosphate $\left(\operatorname{PtdIns}(4,5) \mathrm{P}_{2}\right)$. Transfection with a PtdIns $(4,5) \mathrm{P}_{2}$ probe (based on the plextrin homology $(\mathrm{PH})$ domain of phospholipase $\mathrm{C} \delta$ fused to green fluorescent protein (PLC $\delta$-PH-GFP)) allowed them to track the whereabouts of $\operatorname{PtdIns}(4,5) \mathrm{P}_{2}$ in cells fed with antibody-coated erythrocytes. PtdIns $(4,5) \mathrm{P}_{2}$ accumulated in the phagocytic 'cups' as they extended round the erythrocytes, but then disappeared as soon as engulfment was complete and the vesicle membrane had fused. This was not due to membrane turnover because an acylated GFP that partitions into the inner leaflet of the plasma membrane neither accumulated as much in the phagocytic cup, nor disappeared as rapidly upon vesicle fusion. So can the transient appearance of $\operatorname{PtdIns}(4,5) \mathrm{P}_{2}$ in the phagosome be accounted for by its local production and destruction? Apparently so: immunofluorescence revealed that type $1 \alpha$ phosphatidylinositol 4-phosphate 5-kinase, the enzyme that catalyses the synthesis of $\operatorname{PtdIns}(4,5) \mathrm{P}_{2}$ from PtdIns(4)P, migrates to phagosomes as they form, then dissociates once phagocytosis is complete. But what accounts for the disappearence of PtdIns $(4,5) \mathrm{P}_{2}$ ? This is a tricky question as
PtdIns $(4,5) \mathrm{P}_{2}$ can be either cleaved by PLC or phosphorylated by phosphatidylinositol-3-OH kinase (PI(3)K), and both enzymes have been implicated in phagocytosis. The authors reasoned that PLC $\gamma$ would be involved because activation of the $\mathrm{Fc}$ receptor, which stimulates phagocytosis, also activates PLC $\gamma$. Immunofluorescence with antibodies against PLC 22 supported this hunch, which was then backed up using a probe for one of the products of PLC, diacylglycerol (DAG). This probe, based on the $\mathrm{C} 1$ domain of protein kinase $\mathrm{C} \delta(\mathrm{C} \delta$-GFP), showed that DAG is produced locally at the phagosome. Transfection of the two lipid probes together (one fused to yellow fluorescent protein (C $\delta$-YFP), the other fused to cyan fluorescent protein (PLC $8-\mathrm{PH}$ CFP)) allowed the two lipids to be tracked simultaneously (see picture: 1, early phagocytic cup; 2 , later phagocytic cup; 3 , early phagosome; 4 , mature phagosome): as PLC $\delta$-PH-CFP dissociated from the phagosome, $C \delta$-YFP became associated, reaching a peak during vesicle closure. DAG therefore seems to be produced from PtdIns $(4,5) \mathrm{P}_{2}$ by phospholipase $\mathrm{C}$ at the phagosome.

But are PtdIns $(4,5) \mathrm{P}_{2}$ and DAG needed for phagocytosis? Sequestration or removal of PtdIns $(4,5) \mathrm{P}_{2}$ reduced phagocytosis, as did treatment with PLC inhibitors. The PLC inhibitors prevented macrophages from forming proper phagocytic cups by blocking actin remodelling at sites of erythrocyte attachment.

The next course in this phagocytic feast might explain how changes in the levels of $\operatorname{PtdIns}(4,5) \mathrm{P}_{2}$ and DAG affect cytoskeletal dynamics, to translate regulation into the mechanics of engulfment.

Another important question concerns $\operatorname{PtdIns}(4,5) \mathrm{P}_{2}$ metabolism. Is it all hydrolysed by PLC, or is some of it converted to PtdIns $(3,4,5) \mathrm{P}_{3}$ ? Probes for this lipid might soon provide an answer.

(1) References and links

Cath Brooksbank ORIGINAL RESEARCH PAPER Botelho, R. J. et al. Localized biphasic changes in phosphatidylinositol-4,5-bisphosphate at sites of phagocytosis. J. Cell Biol. 151, 1353-1367 (2000)

Cell Biol. 151, 1353-1367 (2000)
FURTHER READING Kwiatkowska, K. \& Sobota, A. Signalling pathways in phagocytosis.Bioessays 21, 422-431 (1999)
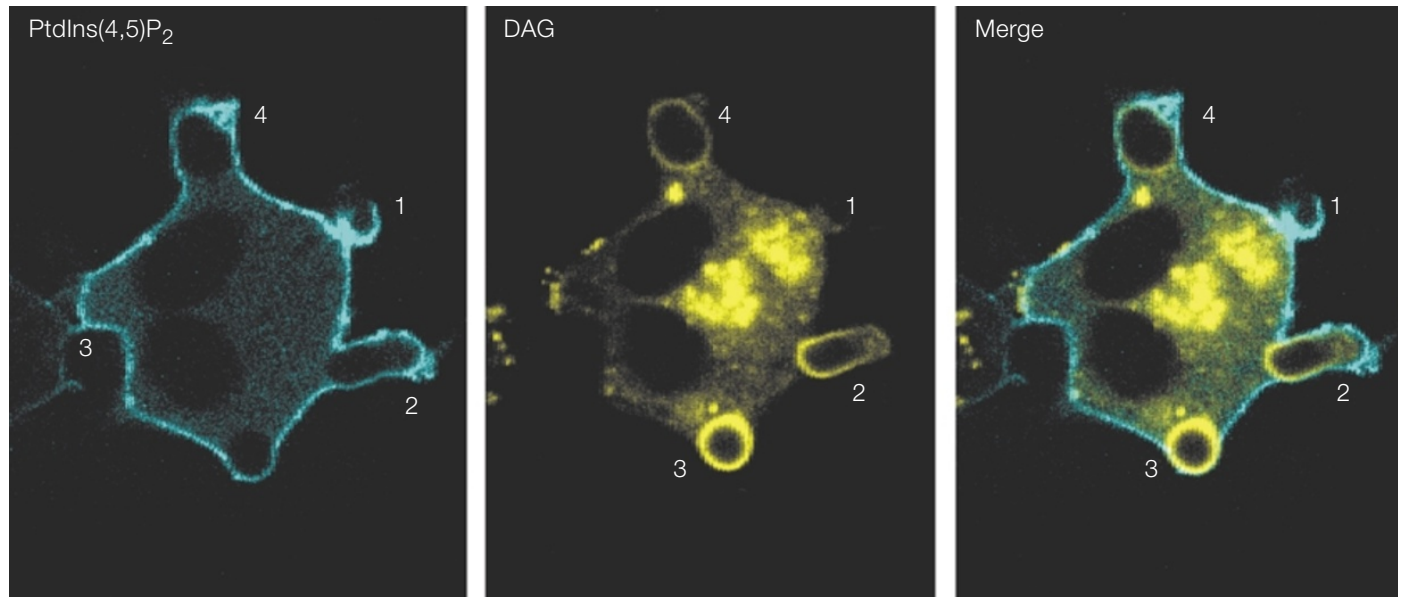

\section{WEB WATCH}

\section{Nuclear fuel}

When is a 'dot com' not a 'dot com'? When it's cellnucleus.com, a new community-driven web site for those interested in the structure and function of the nucleus. The aim is not to make money, but to provide an up-to-date resource for all cell nucleus researchers.

Established and maintained by Michael J. Hendzel from the University of Alberta,

cellnucleus.com was officially launched last December.

"The principal goal ... is to freely disseminate information on the cell nucleus in order to stimulate interest in the topic", says Hendzel.

A core component of the site is an evolving textbook, with several prominent names signed up to contribute chapters. But at the moment, only two 'temporary' reviews are posted. The site also features an antibody database and a list of links to the home pages of various cell nucleus researchers.

One useful tool is a literature update, listing recently published papers in the field. Papers can be viewed by date or by category, and there are links to PubMed. Other features that will be added to the site include job and conference listings, posters and Powerpoint presentations from meetings, as well as a live-cell imaging database. This database should ultimately become a forum for "descriptive observations obtained in livecell experiments that may be of value to the community but do not fit nicely into any manuscripts derived from ongoing research projects within the laboratories involved".

To get to the site at the moment you'll need to go to http://www.cellnucleus.org, but it should move to www.cellnucleus.com around the middle of the year. In the meantime, the site will continue to evolve, and the idea is that it will fuel discussion in the field and provide a useful teaching resource. 